\title{
The association between vascular risk factors and the occurrence of non-thrombotic iliac vein lesions in patients with chronic venous disorders
}

\author{
Radosław Krzyżański, Łukasz Dzieciuchowicz \\ Department of Vascular Surgery and Vascular Diseases, Institute of Medical Sciences, Collegium Medicum, \\ University of Zielona Góra, Poland
}

\begin{abstract}
Introduction: Recently non-thrombotic iliac vein lesions (NIVL) due to their possible role in chronic venous insufficiency on one side and to the development of endovascular venous techniques on the other side have gained much interest. The purpose of this study was to establish if vascular risk and anthropometric factors are associated with the occurrence of NIVL in patients with chronic venous disorders.

Material and methods: Thirty-three patients ( 8 men and 25 women) of the median age of 48 years with primary varicose veins that were qualified for great saphenous veins high ligation and stripping were included. The data concerning age, sex, body mass, height, body mass index, body surface area, hypertension, hypercholesterolemia, smoking and diabetes have been collected. During the varicose vein surgery, both iliac venous axis were interrogated with intravascular ultrasound. Percentage of stenosis of interrogated veins was calculated. The association between analyzed factors and morphology of iliac veins was statistically determined.

Results: In a univariate analysis age negatively correlated with left common iliac vein (LCIV) stenosis and male sex, greater weight and body surface area and hypertension were associated with lesser stenosis of left external iliac vein. In a multivariate analysis, only age significantly negatively correlated with LCIV stenosis $(p=0.027)$. There was a correlation of borderline statistical significance between female sex and LCIV stenosis ( $p=0.073)$. No other correlations were observed.
\end{abstract}

Conclusions: Except for age and possibly sex, there is no association between NIVL and other anthropometric and vascular risk factors.

Key words: intravascular ultrasound, non-thrombotic iliac vein lesions, primary varicose veins

Acta Angiol 2020; 26, 2: 5I-57

\section{Introduction}

Chronic non-thrombotic iliac vein lesions (NIVL) described by May and Turner in 1957 develop as a consequence of compression of iliac vein, predominantly left common iliac vein, between artery and bones [I]. As suspected earlier by Virchov and McMurrich, they may predispose to deep venous thrombosis, but they also may contribute to a wide range of symptoms of chronic venous disorders (CVD) [2, 3]. Since nowadays these lesions can be treated by angioplasty and stenting their diagnosis may have important treatment implications. In distinction to venous reflux or obstruction in below inguinal ligament segment, NIVL are more difficult to diagnose and for that purpose usually more sophisticated and/or invasive tests such as computed tomography, magnetic resonance imaging, contrast phlebography or intravascular ultrasound is required. Taking into account the widespread prevalence of CVD, peaking to $40-50 \%$ of western populations, it is obvious and even inappropriate to perform these tests in all patients with the sign and symptoms of the disease [4-6]. Thus,

Address for correspondence: Radosław Krzyżański, Department of Vascular Surgery and Vascular Diseases, Institute of Medical Sciences, Collegium Medicum, University of Zielona Góra, Zyty 26, 65-046 Zielona Góra, Poland, e-mail: radzinek@gmail.com 
the identification of clinical factors that would point to the possibility of the existence of such lesions would be helpful in the selection of CVD patients for further, more sophisticated imaging tests. Since the principal cause of this lesion is the compression of the iliac veins, most commonly left common iliac vein, between the artery and bone we hypothesized that either factor affecting a body habitus such as demographics and body size or factors affecting behaviour of the arteries such as hypertension, diabetes, hypercholesterolemia or smoking may have an influence on occurrence of NIVL.

The purpose of the study was to determine an association between clinical factors and occurrence and severity of non-thrombotic iliac vein lesions in patients with chronic venous disorders.

\section{Material and methods}

The protocol of the study was approved by the institutional bioethical committee. All of the enrolled patients gave informed consent for participation in the investigation.

The study included patients presenting with unilateral primary varicose veins (PV) associated with great saphenous vein (GSV) incompetence, scheduled for GSV ligation and striping. Before the inclusion, a clinical examination and a duplex Doppler of the lower limb venous system was performed.

The following exclusion criteria have been adopted: history or ultrasound signs of proximal deep venous thrombosis, patients under 18 years of age, pregnancy and breastfeeding, severe chronic venous insufficiency defined as the C4b-C6 class of CEAP classification [7], chronic and acute lower limb ischemia, known thrombophilia or other coagulation disorders, lymphedema, any acute or chronic inflammatory disease, active cancer or history of chemo- or radiotheraphy, symptomatic coronary artery disease, history of major pelvic or retroperitoneal surgery or trauma, aortic or iliac aneurysms or portal hypertension, patients with cirrhosis.

Thirty-three patients, 8 men and 25 women were included. The median age of the patients was $48.2 \pm$ I 3.8 years. Before the procedure, the patients' weight and height were determined and body mass index (BMI) and body surface area (BSA) were calculated. The data concerning following vascular risk factors: hypertension, hypercholesterolemia, smoking and diabetes were collected.

During the varicose vein surgery, an intravenous ultrasound interrogation of inferior vena cava and both right and left common and external iliac veins was performed with Volcano s5 Imaging System (Volcano Corporation, Rancho Cordova, CA, USA) according to the technique described in the literature [8]. In short, through a 9Fr introducer sheath inserted under direct vision into proximal GSV at the operated side and through ultrasound-guided percutaneous puncture at the non-operated side, a Visions PV.035 catheter, with $10 \mathrm{MHz}$ frequency transducer, and the maximum imaging diameter of $60 \mathrm{~mm}$ was advanced over a guidewire, at the level of the right atrium. During the manual pull-back, the veins of interest were interrogated with IVUS and the images obtained were archived on the hard drive of the device and DVD.

The morphometric analysis was carried out on the Volcano s5 Imaging System, using the standard software installed on the device. Inferior vena cava (IVC) and both, left and right, common iliac veins (CIV) and external iliac veins (EIV) were measured. In each analyzed vein cross-sectional area (CSA) of a non-stenotic segment of the vessel and of the most stenosed segment were determined and denominated a reference cross-sectional area (ref-CSA) and a Minimal Lumen Area (MLA), respectively (Fig. I). While determining the ref-CSA the areas of venous confluence were excluded from the measurement. Percentage of stenosis (S\%) of each analyzed vessel was determined according to the following formula:

$$
S \%=(\text { ref-CSA }- \text { MLA }) / \text { ref-CSA } \times 100
$$

In the further analysis, the influence of the vascular risk factors, anthropometric and demographic factors on the morphological parameters of examined veins were studied.

\section{Statistical analysis}

- For quantitative variables mean, SD, median, quartiles and range were shown.

- For qualitative variables, absolute and percentage distributions were shown.

- Quantitative variables were compared between two groups by the means of t-Student (in case of normality of distribution in both groups) and Mann-Whitney test (otherwise).

- Correlations between quantitative variables were analyzed with Spearman's correlation coefficient.

- Normality was checked with the Shapiro-Wilk test.

- A linear regression model was used for a multivariate analysis.

- Significance level was set at 0,05.

- Analysis was conducted in R package, version 3.2.3.

\section{Results}

The procedure was carried out according to the protocol in all included patients. Fourteen (43\%) of the thirty-three patients enrolled in the study had 


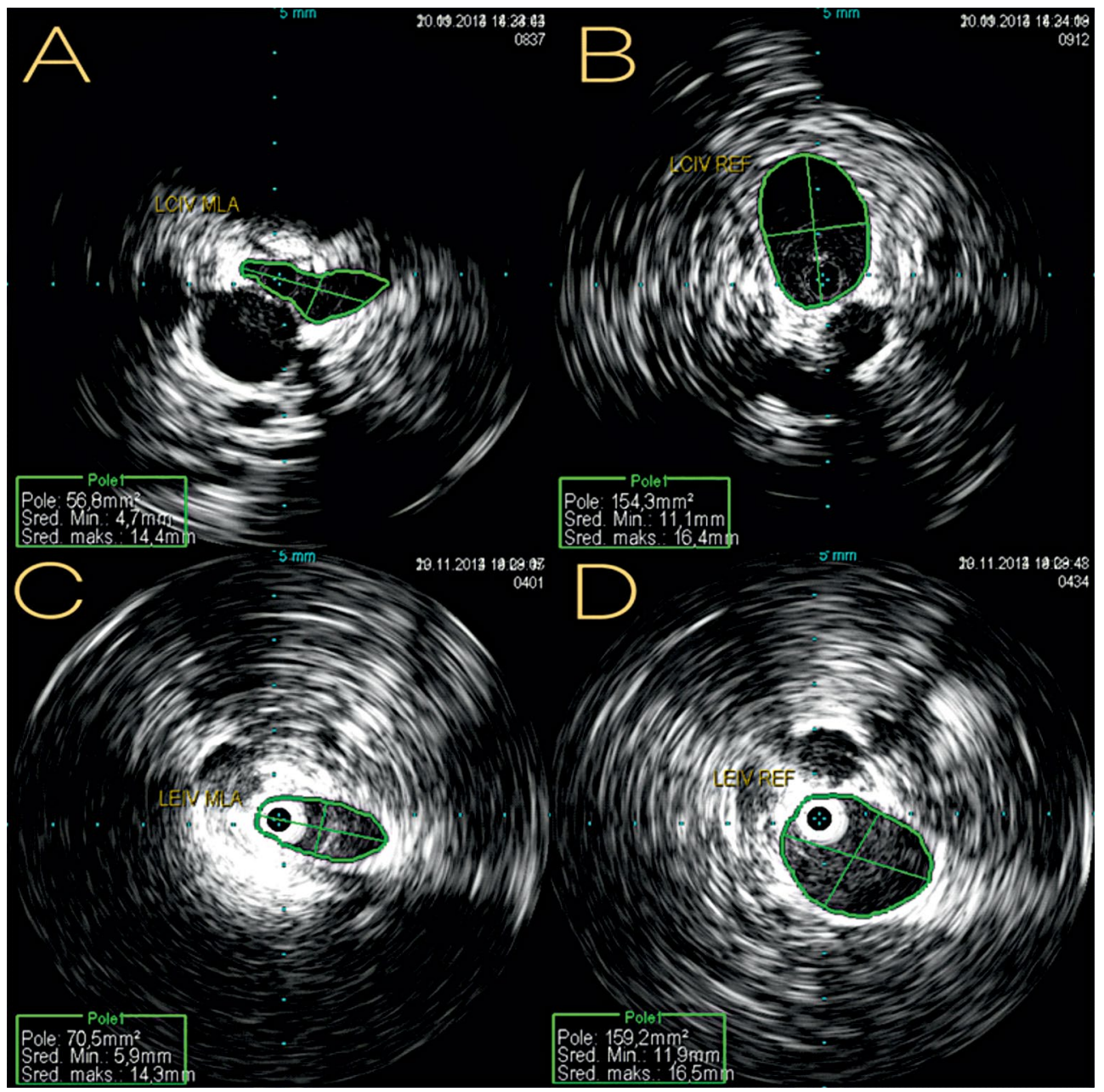

Figure I. An intravascular ultrasound (IVUS) images of left common iliac vein (LCIV) and external iliac vein (LEIV) showing a minimal lumen area (MLA) (panels $A$ and $C$ ) and a reference cross-sectional area (REF) (panels $B$ and $D)$

varicose veins in the left lower limb. The mean height, body weight, body mass index (BMI) and body surface area (BSA) were I $70(9.2) \mathrm{cm}, 79$ (I3.2) kg, 27.3(4.2) $\mathrm{kg} / \mathrm{m}^{2}$ and $\mathrm{l} .9(0.2) \mathrm{m}^{2}$, respectively. The prevalence of vascular risk factors is presented in Table I. The median values of $\mathrm{S} \%$ of the examined veins limb were 68.65 (48.43-78.99) \% for LCIV, 45.47 (38.03-57.8) \% for LEIV, 34.45 (24.44-48. I8) \% for RCIV and 45.24 (36.05-57.42) \% for REIV.

In the univariate analysis with regard to common iliac veins, there was a statistically significant, moderate and negative correlation between LCIV stenosis and age of the patients (Fig. 2). None of the other analyzed anthropometrics and vascular risk factors had a statistically significant influence on the stenosis of common iliac veins. Since none of the patients had diabetes its influence could not be analyzed. The details are presented in Tables 2 and 3. With regard to external iliac veins male sex and the presence of hypertension was related to lesser LEIV stenosis and there was a statis-
Table.I. The prevalence of vascular risk factors in the examined group of patients

\begin{tabular}{|l|c|c|c|}
\hline Clinical factor & \multicolumn{1}{c}{$\begin{array}{c}\text { All } \\
\text { patients } \\
\text { n (\%) }\end{array}$} & $\begin{array}{c}\text { Female } \\
\text { group } \\
\text { n (\%) }\end{array}$ & $\begin{array}{c}\text { Male } \\
\text { group } \\
\text { n (\%) }\end{array}$ \\
\hline Hypertension & $6(18.18)$ & $3(12)$ & $3(37.5)$ \\
\hline Hypercholesterolemia & $4(12.1)$ & $3(12)$ & $1(12.5)$ \\
\hline Smokers & $10(30.30)$ & $8(32)$ & $2(25)$ \\
\hline Diabetes & $0(0)$ & $0(0)$ & $0(0)$ \\
\hline
\end{tabular}

tically significant, moderate and negative correlation between LEIV stenosis and weight and BSA (Figs 3, 4). None of the other analyzed anthropometrics and vascular risk factors had a statistically significant influence on the stenosis of external iliac veins. The details are presented in Tables 3 and 4.

The multivariate analysis confirmed a statistically significant, negative correlation between LCIV and age 


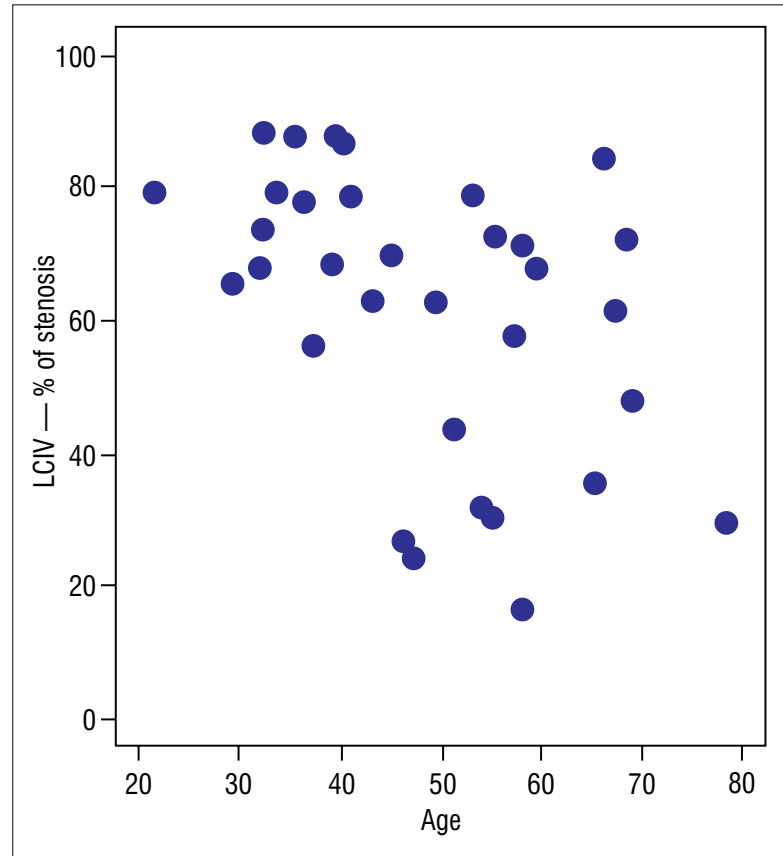

Figure 2. A scatterplot presenting a correlation between the percentage of stenosis of left common iliac vein (LCIV) and age of the patients. Spearman coefficient $r=-0.47, p=0.0$ I

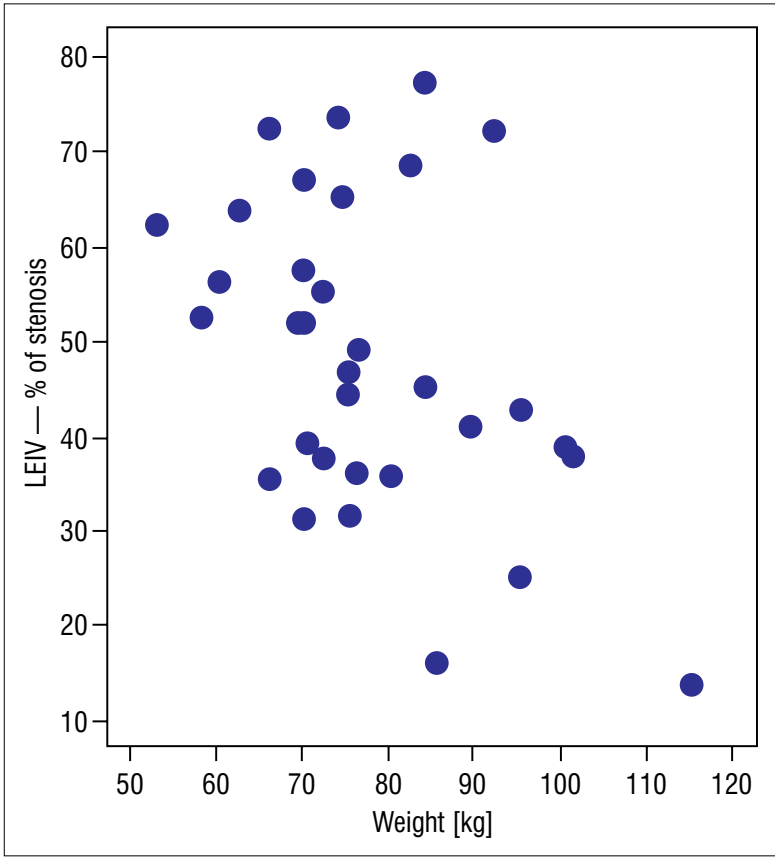

Figure 3. A scatterplot presenting a correlation between the percentage of stenosis of left external iliac vein (LEIV) and weight of the patients. Spearman coefficient $r=-0.37, p=0.04$

Table 2. Stenosis of the left ( $\mathrm{LCIV})$ and right (RCIV) common iliac veins in relation to the presence of vascular risk factors

\begin{tabular}{|c|c|c|c|c|}
\hline & \multicolumn{2}{|c|}{ LCIV - \% S } & \multicolumn{2}{|c|}{ RCIV - \% S } \\
\hline & Median (QI-Q3) & $\mathbf{p}$ & Median (QI-Q3) & $\mathbf{p}$ \\
\hline Men & $60.54(53.49-69.08)$ & \multirow{2}{*}{$p=0.254$} & $32.84(26.98-42.52)$ & \multirow{2}{*}{$p=0.984$} \\
\hline Women & $70.16(48.43-79.47)$ & & $34.45(21.5-49.76)$ & \\
\hline Hypertension & $59.86(41.69-69.09)$ & \multirow{2}{*}{$p=0.398$} & $37.17(16.77-49.98)$ & \multirow{2}{*}{$p=0.838$} \\
\hline No hypertension & $68.93(52.55-79.12)$ & & $34.45(24.62-46.45)$ & \\
\hline Hypercholesterolemia & $71.69(68.08-74.67)$ & \multirow{2}{*}{$\mathrm{p}=0.477$} & 15.61 (1 I.44-29.22) & \multirow{2}{*}{$p=0.183$} \\
\hline No hypercholesterolemia & $67.11(47.31-74.45)$ & & $31.39(26.22-44.64)$ & \\
\hline Smokers & $65.96(56.67-78.09)$ & \multirow{2}{*}{$p=0.432$} & $40.58(26.69-44.7 I)$ & \multirow{2}{*}{$p=0.967$} \\
\hline No smokers & $71.5 ।$ (6I.83-79.47) & & 34.45 (24.79-49.76) & \\
\hline
\end{tabular}

Table 3. Correlation of stenosis of left common iliac vein (LCIV), left external iliac vein (LEIV), right common iliac vein (RCIV) and right external iliac vein (REIV) with anthropometrics

\begin{tabular}{|c|c|c|c|c|c|c|c|c|}
\hline \multirow[t]{2}{*}{ Correation with } & \multicolumn{2}{|c|}{ LCIV - \% S } & \multicolumn{2}{|c|}{ LEIV - \% S } & \multicolumn{2}{|c|}{ RCIV - \% S } & \multicolumn{2}{|c|}{ REIV - \% S } \\
\hline & Coefficient & $\mathbf{p}$ & Coefficient & $\mathbf{p}$ & Coefficient & $\mathbf{p}$ & Coefficient & $\mathbf{p}$ \\
\hline Age & -0.466 & 0.006 & -0.258 & 0.147 & -0.08 & 0.656 & -0.048 & 0.79 \\
\hline height & 0.16 & 0.374 & -0.228 & 0.202 & -0.019 & 0.917 & 0.283 & 0.111 \\
\hline weight & 0.109 & 0.545 & -0.365 & 0.036 & 0.03 & 0.869 & 0.046 & 0.801 \\
\hline $\mathrm{BMI}$ & -0.028 & 0.875 & -0.234 & 0.189 & -0.102 & 0.572 & 0.01 & 0.956 \\
\hline BSA & 0.133 & 0.459 & -0.377 & 0.031 & 0.036 & 0.842 & 0.097 & 0.592 \\
\hline
\end{tabular}




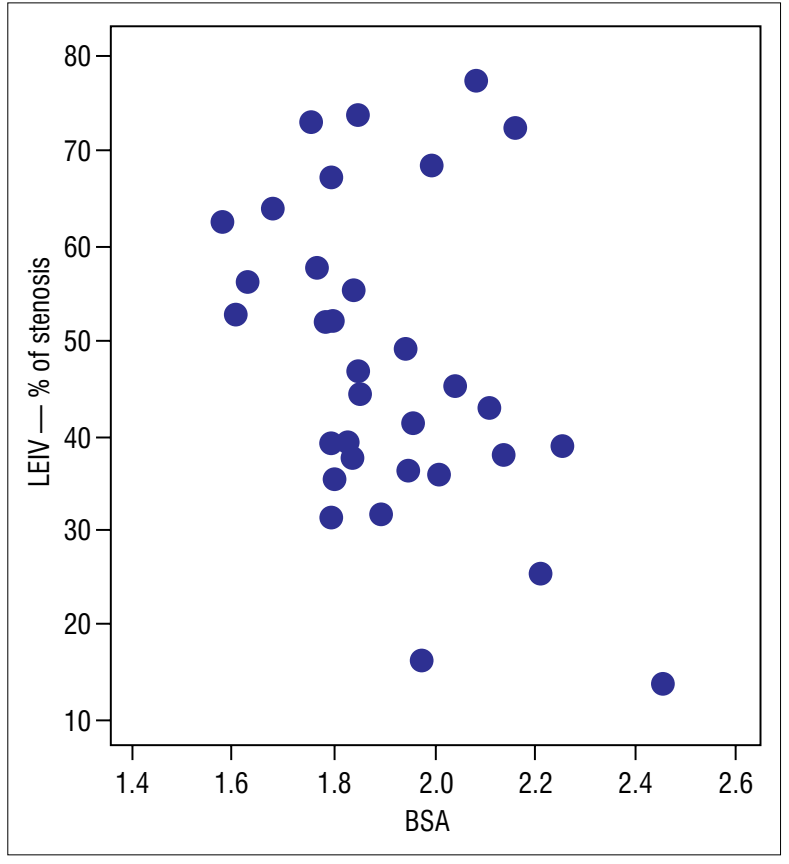

Figure 4. A scatterplot presenting a correlation between the percentage of stenosis of left external iliac vein (LEIV) and body surface area (BSA) of the patients. Spearman coefficient $r=-0.38, p=0.03$ $(p=0.029)$. There was also a borderline statistical significance $(p=0.073)$ trend towards greater LCIV stenosis in women. No other statistically significant correlations were observed. The details are presented in Table 5.

\section{Discussion}

To the best of our knowledge, this is the first published study that analyzed the association between anthropometrics and vascular risk factors and the occurrence of non-thrombotic iliac vein stenosis. To determine the presence and severity of iliac vein stenoses an intravascular ultrasound was used that is considered the most sensitive modality to diagnose these lesions [9-12]. The main findings of this study are that except for age and possibly gender there are not relations between non-thrombotic iliac veins stenosis and vascular risk and anthropometric factors. The multivariate analysis confirmed a negative correlation between LCIV and age There was also a borderline statistical significance trend towards greater LCIV stenosis in women. With regard to LEIV though the univariate analysis has shown correlations between male sex, hypertension, greater

Table 4. Stenosis of left (LEIV) and right (REIV) external iliac veins in relation to the presence of vascular risk factors

\begin{tabular}{|c|c|c|c|c|}
\hline & \multicolumn{2}{|c|}{ LEIV - \% S } & \multicolumn{2}{|c|}{ REIV - \% S } \\
\hline & Median (QI-Q3) & $\mathbf{p}$ & Median (QI-Q3) & $\mathbf{p}$ \\
\hline Men & $37.23(33.64-40.86)$ & \multirow{2}{*}{$p=0.036$} & $51.73(42.9-56.54)$ & \multirow{2}{*}{$p=0.445$} \\
\hline Women & $52.23(39.56-62.68)$ & & $44.1(33.01-59.06)$ & \\
\hline Hypertension & $37.23(21.55-38.28)$ & \multirow{2}{*}{$p=0.027$} & $45.39(38.66-51.67)$ & \multirow{2}{*}{$p=0.633$} \\
\hline No hypertension & $49.31(39.54-63.45)$ & & $45.24(35.72-58.98)$ & \\
\hline Hypercholesterolemia & $37.48(27.9-50.64)$ & \multirow{2}{*}{$p=0.477$} & $46.8(36.75-58)$ & \multirow{2}{*}{$p=0.794$} \\
\hline No hypercholesterolemia & $46.2(37.88-58.12)$ & & $41.5(32.88-58.94)$ & \\
\hline Smokers & $46.93(38.36-53.13)$ & \multirow{2}{*}{$p=1$} & $54.46(38.33-56.98)$ & \multirow{2}{*}{$p=0.483$} \\
\hline No smokers & $45.47(36.28-62.68)$ & & $42.53(36.05-58.9)$ & \\
\hline
\end{tabular}

Table 5. Linear regression model of the influence of vascular risk factors and anthropometrics on the stenosis (S\%) of LCIV and LEIV in the analyzed group of patients

\begin{tabular}{|c|c|c|c|c|c|c|c|c|}
\hline & \multicolumn{4}{|c|}{ LCIV - \% S } & \multicolumn{4}{|c|}{ LEIV- \% S } \\
\hline & Coefficient & SE & $\mathbf{t}$ & $\mathbf{p}$ & Coefficient & SE & $\mathbf{t}$ & $\mathbf{p}$ \\
\hline Female sex (Males as reference) & 20.854 & 11.192 & 1.863 & $p=0.073$ & 5.921 & 8.955 & 0.661 & $p=0.514$ \\
\hline Age [years] & -0.725 & 0.315 & -2.299 & $p=0.029$ & -0.18 & 0.252 & -0.713 & $p=0.482$ \\
\hline Weight $[\mathrm{kg}]$ & -1.178 & 1.299 & -0.907 & $p=0.372$ & -0.609 & 1.039 & -0.586 & $p=0.563$ \\
\hline BSA & 121.685 & 96.656 & 1.259 & $p=0.219$ & 19.772 & 77.334 & 0.256 & $p=0.8$ \\
\hline Hypertension & 11.793 & 11.479 & 1.027 & $p=0.313$ & -7.874 & 9.184 & -0.857 & $p=0.399$ \\
\hline
\end{tabular}


weight and greater BSA and lesser LEIV stenosis, these correlations were not confirmed in the multivariate analysis which means that none of these factors was an independent predictor of LEIV stenosis. Since women weigh less, are shorter and are less frequently hypertensive then men it might have pointed to a tendency towards greater LEIV stenosis in them. However further studies are required to confirm this speculation.

The negative correlation between age and left iliac vein stenosis hasbeen previously observed. In a study of 50 consecutive abdominal computed tomography scans performed for abdominal pain a moderate negative correlation between left iliac vein compression and age, the patients were found [13]. The question arises how this negative correlation between LCIV stenosis and age that at first glance seems counterintuitive can be explained. One would think that with age the arteries become wider, stiffer and more calcified and the vertebral column more deformed that would increase the compression between these structures. However, this not the case. The diameter of iliac artery increases with age, but it does not increase the compression of LCIV [I3]. And as it has been shown in the present study the atherosclerotic risk factors such as hypertension, hypercholesterolemia and smoking are not associated with iliac vein compression. Interesting data comes from the study that compared iliac vein compression between 100 patients with abdominal aortic aneurysm with 100 patients without an abdominal aneurysm. The iliac vein compression was significantly decreased in patients with abdominal aortic aneurysm [14]. The authors of that study contributed to this finding to increased tortuosity of the iliac arteries. But the fact is that the patients without abdominal aortic aneurysm were significantly younger than those with aneurysm what could be associated with greater compression of the iliac vein.

The most probable explanation of decreasing of iliac vein compression with age is a change in the geometry of the spine. It has been demonstrated that together with age there is a loss of lumbar lordosis [15, 16]. And this phenomenon may increase the distance between vertebral column and iliac artery thus decreasing the compression of iliac vein.

The greater LCIV compression in women was also documented in the aforementioned study of 50 consecutive abdominal computed tomography scans [13]. Women also prevail in the groups of patients undergoing stenting of the iliac vein [17]. It was also observed that lordosis is greater in women what may explain the observed trend towards greater LCIV compression $[18,19]$.

In conclusion, the younger age remains the only proved clinical factor associated with non-thrombotic iliac vein compression. Further studies are required to determine the mechanism of this association and the influence of gender.

\section{Conflict of interest}

None.

\section{References:}

I. May R, Thurner J. The Cause of the predominantly sinistral occurrence of thrombosis of the pelvic veins. Angiology. 2016; 8(5): 419-427, doi: 10.1177/000331975700800505.

2. Virchow R. Uber die Erweiterung kleiner Gefasse. Arch Path Anat. 1851; 3: 427.

3. MCMURRICH J. The occurrence of congenital adhesions in the common iliac veins and their relation to the thrombosis of the femoral and iliac veins. Am J Med Sci. 1908; 135(3): 342-345, doi: 10.1097/0000044|-190803000-00004.

4. Carpentier PH, Maricq HR, Biro C, et al. Prevalence, risk factors, and clinical patterns of chronic venous disorders of lower limbs: a population-based study in France. J Vasc Surg. 2004; 40(4): 650-659, doi: 10.1016/j.jvs.2004.07.025, indexed in Pubmed: I547259।.

5. Sisto T, Reunanen A, Laurikka J, et al. Prevalence and risk factors of varicose veins in lower extremities: mini-Finland health survey. Eur J Surg. 1995; 161(6): 405-414, indexed in Pubmed: 7548376.

6. Lee AJ, Robertson LA, Boghossian SM, et al. Prevalence of venous reflux in the general population on duplex scanning: the Edinburgh vein study. J Vasc Surg. 1998; 28(5): 767-776, doi: 10.1016/s074I-52 I4(98)7005 I-5, indexed in Pubmed: 9808843.

7. Eklöf Bo, Rutherford R, Bergan J, et al. Revision of the CEAP classification for chronic venous disorders: Consensus statement. Journal of Vascular Surgery. 2004; 40(6): 1248-1252, doi: 10.1016/j.jvs.2004.09.027.

8. Dzieciuchowicz $Ł$, Krzyżański R, Kruszyna $Ł$, et al. Prevalence of non-thrombotic iliac vein lesions in patients with unilateral primary varicose veins. Eur J Vasc Endovasc Surg. 2016; 51 (3): 429-433, doi: 10.1016/j.ejvs.2015.11.012, indexed in Pubmed: 26739803.

9. Hingorani A, Alhabouni S, Ascher E, et al. Role of IVUS versus venograms in assessment of iliac-femoral vein stenosis. Journal of Vascular Surgery. 2010; 52(3): 804, doi: 10.1016/j. jvs.2010.06.029.

10. Neglén P, Raju S. Intravascular ultrasound scan evaluation of the obstructed vein. J Vasc Surg. 2002; 35(4): 694-700, doi: 10.1067/mva.2002.121127, indexed in Pubmed: I1932665.

II. Montminy M, Thomasson J, Tanaka G, et al. A comparison between intravascular ultrasound and venography in identifying key parameters essential for iliac vein stenting. Journal of Vascular Surgery: Venous and Lymphatic Disorders. 2019; 7(6): 80I-807, doi: 10.1016/j.jvsv.2019.03.015.

12. Gagne PJ, Tahara RW, Fastabend CP, et al. Venography versus intravascular ultrasound for diagnosing and treating iliofemoral vein obstruction. J Vasc Surg Venous Lymphat Disord. 2017; 5(5): 678-687, doi: 10.1016/j.jvsv.2017.04.007, indexed in Pubmed: 28818221 . 
13. Kibbe MR, Ujiki M, Goodwin AL, et al. lliac vein compression in an asymptomatic patient population. J Vasc Surg. 2004; 39(5): 937-943.

14. Moreland NC, Ujiki M, Matsumura JS, et al. Decreased incidence of left common iliac vein compression in patients with abdominal aortic aneurysms. J Vasc Surg. 2006; 44(3): 595-600, doi: 10.1016/j.jvs.2006.05.046, indexed in Pubmed: 16950440.

15. Dreischarf M, Albiol L, Rohlmann A, et al. Age-related loss of lumbar spinal lordosis and mobility--a study of 323 asymptomatic volunteers. PLoS One. 2014; 9(12): el I6186, doi: 10.1371/ journal.pone.0116186, indexed in Pubmed: 25549085.

16. Pries E, Dreischarf M, Bashkuev M, et al. The effects of age and gender on the lumbopelvic rhythm in the sagittal plane in 309 subjects. J Biomech. 2015; 48(12): 3080-3087, doi: 10.1016/j. jbiomech.2015.07.030, indexed in Pubmed: 26294355.

17. Raju S, Neglen P. High prevalence of nonthrombotic iliac vein lesions in chronic venous disease: a permissive role in pathogenicity. J Vasc Surg. 2006; 44(I): 136-43; discussion I44, doi: 10.1016/j.jvs.2006.02.065, indexed in Pubmed: 16828437.

18. Arshad R, Pan F, Reitmaier S, et al. Effect of age and sex on lumbar lordosis and the range of motion. A systematic review and meta-analysis. J Biomech. 2019; 82: I-19, doi: 10.1016/j. jbiomech.2018.1 I.022, indexed in Pubmed: 30503255.

19. Leven D, Merrill R, Kim J, et al. Fundamental differences and changes in pelvic parameters depending on age, gender and race. The Spine Journal. 2016; 16(10): S277, doi: 10.1016/j. spinee.2016.07.386. 